\title{
Single Session Rehabilitation Counseling
}

\author{
A Session in Hand, may be Worth Many in Oblivion!
}

\author{
Geetha Desai - Sivakumar Thanapal · \\ Sailaxmi Gandhi · Nirmala P. Berigai · \\ Santosh K. Chaturvedi
}

Received: 22 November 2014/ Accepted: 22 December 2014/Published online: 25 February 2015

(C) Springer India Pvt. Ltd. 2015

\begin{abstract}
Rehabilitation services are considered essential in the management of mental illnesses. Introduction to these services to the patients and families are highly variable. Single session rehabilitation counseling emerged as a concept based on our observation that many patients and families would have only one contact with the team. The article describes the objectives and methods of single session rehabilitation counseling.
\end{abstract}

Keywords Single session - Counseling - Rehabilitation

\section{Single Session Rehabilitation Counseling}

Rehabilitation/recovery based services are considered essential in the management of mental illnesses. The awareness related to these services is abysmal in caregivers, patients and even professionals. The term rehabilitation often is misconstrued as a last resort for patients who do not get better with treatment and/or are treatment failures. Hence, the concept of recovery could be a better replacement for the concept of rehabilitation. Mental health professionals have a varied opinion on timing of introducing

G. Desai $(\bowtie) \cdot$ S. Thanapal · S. K. Chaturvedi

Department of Psychiatry, National Institute of Mental Health and Neurosciences, Bengaluru, Karnataka, India

e-mail: desaigeetha@gmail.com

\section{S. Gandhi}

Department of Nursing, National Institute of Mental Health and Neurosciences, Bengaluru, Karnataka, India

N. P. Berigai

Department of Psychiatric Social Work, National Institute of Mental Health and Neurosciences, Bengaluru, Karnataka, India this concept of recovery to families and patients. Most mental health professionals know that long term outcome of patients with psychiatric disorders is likely to be better with rehabilitative interventions. However, there could be prolonged delays in introducing recovery services to patients and families. Hence, it is important to discuss these aspects with patients and their families, as and when possible.

As with the professionals, rehabilitation services do not seem to be very popular or sought after by many persons with mental illnesses or their families. Probably such services are low in the priority of interventions, as compared to medications. In a study on first intake assessments of patients who were referred from inpatients services, the duration of stay and the number of days the patients attended rehabilitation services was found to be highly variable from one day to few weeks [1]. There is a substantial gap in the patient and mental health professional ratio with significant shortage of trained mental health professionals. Given this scenario, the chances of only a single consultation is quite high and the luxury of having multiple sessions may be a mirage.

Erna Hoch in her seminal work on psychotherapy in traditional milieu noticed that often patients would have only one consultation. She describes the methods of making the first consultation very effective [2]. In this background, where the concept of rehabilitation is unfamiliar and the duration of stay is highly variable single session rehabilitation counseling could be a viable option.

The objectives of this single session rehabilitation counseling can be

- To understand the awareness among caregivers about rehabilitation.

- To provide information about rehabilitation services. 
- To assess their available resources.

- To assess the abilities of the patients.

- To generate a plan for rehabilitation.

The first step of the process is to understand the concept of rehabilitation from the patient and caregivers perspective. This also includes eliciting myths and misconceptions related to recovery. This also provides information on their understanding about the illness and treatment and their expectations from the patient and the expectations of patients from themselves. This also explores their knowledge of availability of services.

The second step in the process is to provide information on various rehabilitation services and facilities available not only at their current hospital but also to provide information of services available at the places where the patient is likely to reside. These services could be institutional, community based, home based, or those supported by non governmental organisations.

The next step in the process is to assess the resources that the family or the communities in which the family and the patient stay, so as to utilize these resources for providing services. This also helps in utilizing the existing resources to the maximum potential. Assessing the patient's potential and abilities is most crucial in planning rehabilitation services. This includes the factors that are potentially interfering and facilitating rehabilitation namely illness status, treatment difficulties, disability and also education and employment.

At the end of this process a possible rehabilitation plan is generated using the information that has been obtained and the feedback is provided to the patient and family member. This could also include the possibility of engaging the person and family for future interventions.

The session is a time limited process. The advantages are that there is limited resource use, and seeding information in a single session could avoid losing out on caregiver questions. Such a method can be useful in busy centers, where there is a shortage of staff, or time is limited. However, there are challenges for the same, namely, patient being symptomatic, caregiver burden leading to difficulty in participating in the session, difficult circumstances in the family, non availability of services in particular region. There may be certain situations when a single session counseling may not be satisfactory enough, for example, if expressed emotions need to be addressed, the first session is useful in setting the agenda and discussing the plan of interventions. Hence, the single session is not a panacea for all the problems or issues related to rehabilitation, but it ensures that most persons are provided with important information which would be helpful in the recovery process. It raises issues about 'something is better than nothing' versus 'some information may cause some damage than do any good'. Hence, the efficacy of this single session counseling, needs to studied by an appropriate research design.

There can be potential variations for this single session method, like having a group single session, or having a single session addressed by a team.

Training in such single session rehabilitation counseling can be done by using role play methods, or using videos or video feedbacks.

The components of the single session counseling are provided below in the box.

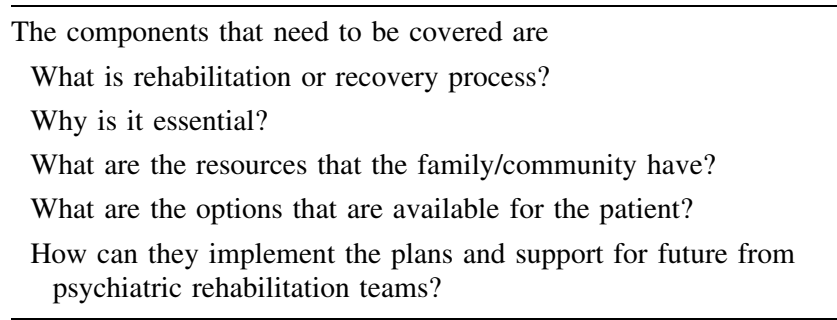

Single session rehabilitation counseling in terms of teaching integrated with service is beneficial in places where there is a floating (less hours of rehabilitation training) population of trainees. The benefits are more tangible especially when the prospect of continuity of services by trainees is quite bleak. As teaching is integrated with service, involvement of trainees in single session rehabilitation counseling will be a double edged sword in terms of resource utilization as well as delivering supervised service. In this time span, consultants can engage in single session flash mentoring to guide or support trainees in their learning experience. The single session rehabilitation counseling offers much for crosssectional quantitative research as well. Evaluation studies as well as follow up of outcome assessment can be done too. Single session rehabilitation counselling can be delivered to remote areas through tele-counseling services. Trainees at both ends of the tele-counseling site can be involved in this as active team members hence professionals can strengthen resources in rural and remote areas too.

To conclude, single session rehabilitation counseling is a challenge in the overly-crowded clinics in India where mental health issues are on the surge. However, it is worth trying out and may even turn out to be an asset in this country where there is a lamentable paucity of mental health professionals. It is to be considered a structured intervention rather than a mere counseling session.

This method is inspired by the challenges of extremely high drop outs of persons with mental illness and 
their caregivers, who could afford, time wise, only one encounter with the rehabilitation team. In order to make the most of this single interaction, this single session rehabilitation counseling is being used by the authors and their team. This method is not limited to the rehabilitation teams but to all the clinicians who need to include rehabilitation as a part of their management plans for patients.

\section{References}

1. Desai G, Narasimha A, Harihara SN, Dashrath MS, Bhola P, Berigai PN, et al. A study on first intake assessments of in-patient referrals to psychiatric rehabilitation services. Indian J Psychol Med. 2014;36(3):236-8.

2. Hoch ME. Psychotherapy for the illiterate, pp 75-92. In: Arieti S, Chrzanowski G, editors. A new dimension in psychiatry, a world view. New York: Wiley; 1977. 